\title{
Correlation of Walking Speed with Pelvic and Lower Limb Movements of Healthy Young Males
}

\author{
TAKASHI NISHIMORI, RPT, MSc ${ }^{1)}$, AKIRA ITO, $\mathrm{PhD}^{2)}$ \\ 1) Department of Physical Therapy, Kansai Vocational College of Medicine: \\ 6-18-13 Karita, Sumiyoshi-ku, Osaka 558-0011, Japan. TEL: +81 6-6699-2222, FAX: +81 6-6699-5335, \\ E-mail:nisimori@kansai.ac.jp \\ ${ }^{2)}$ Laboratory of Sport Biomechanics, Graduate School, Osaka University of Health and Sports Sciences
}

\begin{abstract}
Purpose] The present study investigated whether moving the front extremity forward over the center of mass at the moment of initial contact or moving the rear extremity backward at the moment of preswing affects the elongation of step length. [Subjects and Methods] Step length can be subdivided into touch down distance (TD), release distance (RD) and displacement during the double stance phase (DD). The subjects were 6 healthy men. The target walking speeds were $1.3 \mathrm{~m} / \mathrm{s}, 1.9 \mathrm{~m} / \mathrm{s}$, and $2.5 \mathrm{~m} / \mathrm{s}$. Walking was recorded using three high-speed cameras. [Results] Step length increased linearly with walking speed, showing a significant relationship ( $\mathrm{r}=0.74)$. TD increased as walking speed rose from $1.3 \mathrm{~m} / \mathrm{s}$ to $1.9 \mathrm{~m} / \mathrm{s}$, then decreased after $1.9 \mathrm{~m} / \mathrm{s}$. No significant correlations were found between step length and TD. RD demonstrated a significant positive correlation with step length $(\mathrm{r}=0.50)$ and also showed significant positive correlations with external pelvic rotation angle $(\mathrm{r}=0.56)$, hip extension angle $(\mathrm{r}=0.61)$, and ankle plantar flexion angle $(\mathrm{r}=0.50)$ at the moment of foot release. [Conclusion] These results indicate that moving the rear extremity backward at the moment of foot release can thus be reasonably be expected to cause elongation of step length.
\end{abstract}

Key words: Touch down distance, Release distance, Three-dimensional analysis

(This article was submitted Jun. 16, 2011, and was accepted Jul. 12, 2011)

\section{INTRODUCTION}

Walking is the fundamental mode of locomotion for humans, and is regulated depending on the environment and the activities being undertaken. Walking is thus performed at a variable speed rather than at a constant speed.

Walking speed is the product of step length and step frequency ${ }^{1-4)}$. Previous reports ${ }^{1,2,5,6)}$ have shown that step length and step frequency show significant linear increases with walking speed. Zatsiorky et al. ${ }^{4)}$ reported that the magnitude of step length is controlled via a seven-segment kinematic chain, such as feet, shanks, thighs, and pelvis with at least 12 degrees of freedom, since step length is approximately the base of a triangle formed by the two limbs at the moment of foot touch down and foot release.

Although several authors ${ }^{1,7-11)}$ have suggested that moving the front extremity forward leads to an elongation of step length, objective evidence of this has been lacking, and, the distance between touch down and center of mass has not been calculated in previous studies.

Novacheck ${ }^{9)}$, Perry ${ }^{10)}$ and Bruijn et al. ${ }^{12)}$ suggested that internal pelvic rotation (pelvis with swing side rotating forward) during the swing phase causes elongation of the distance between the point of foot touch down and center of mass, representing touch down distance (TD), but did not actually calculate the TD. Inman et al. ${ }^{13)}$ only presented objective data showing a significant positive correlation between amplitude of pelvic rotation angle and step length. However, this is not clear proof that TD causes the elongation of step length. In relation to the lower extremity, angles of the hip, knee, and ankle at the moment of touch down have shown inconsistent increases in relation to walking speed ${ }^{5,8,11)}$, and the investigated walking speeds were below $2.0 \mathrm{~m} / \mathrm{s}^{11-13)}$. How the pelvis and lower extremity joints interact to lengthen step length thus remains unclear. The present study investigated up to $2.5 \mathrm{~m} /$ s walking speed.

The purpose of the present study was to investigate whether moving the front extremity forward over the center of mass at the moment of foot contact or moving the rear extremity backward at the moment of foot release affects the elongation of step length in relation to walking speeds up to $2.5 \mathrm{~m} / \mathrm{s}$. To achieve this purpose, we calculated the center of mass at the moment of foot touch down and foot release, then measured the TD and the distance from the center of mass to the point of foot release at the moment of foot release (release distance: RD), using three-dimensional motion analysis. In addition, the present study measured angles of pelvic rotation in the transverse plane and angles of the hip, knee, and ankle in the sagittal plane as components of TD and RD. Furthermore, the overall roles of the pelvis and lower extremity in lengthening TD and $\mathrm{RD}$ were investigated. The results of this study should be useful for the instruction of walking practice in physical 
therapy and the understanding of compensated walking.

\section{SUBJECTS AND METHODS}

The subjects were 6 healthy men (mean age, $19.7 \pm 0.52$ years; height, $169.1 \pm 8.66 \mathrm{~cm}$; body mass, $63.5 \pm 6.31 \mathrm{~kg}$ ). Subjects wore their own running shoes (no spikes), running semi-tights adhered to the pelvic girdle, and no top. All subjects provided their written informed consent before participation in the study, and all study protocols were approved by the ethics committee of the Graduate School of Sport and Exercise Science of Osaka University of Health and Sports Sciences. No subjects experienced any injuries during this experiment.

Subjects were videotaped using three high-speed cameras at a sampling rate of $100 \mathrm{~Hz}$ while walking on an all-weather track (Memrecam $\mathrm{c}^{2} \mathrm{~s}$; NAC, Japan). The optical axis between each camera was about 120 degree. An adjustable-speed pacemaker was set along the 40-m straight track. Each subject walked while visually watching a moving flag synchronized to the pacemaker. The paced walking speeds set were $1.3,1.9$ and $2.5 \mathrm{~m} / \mathrm{s}$. The actual walking speeds utilized in this study were calculated by motion analysis.

Three-dimensional coordinates were calculated using the direct linear transformation method ${ }^{14)}$. Twenty-six body landmarks were digitized using Frame-DIAS II software (DKH, Japan), identifying in each frame the landmark being visually monitored on a desktop computer. Digitized landmarks were parietal, the midpoint between the auricles, sternum, center of the shoulder joint (right (Rt) and left $(\mathrm{Lt})$ ), center of the elbow joint (Rt and Lt), center of the wrist joint (Rt and Lt), metacarpophalangeal joint (Rt and $\mathrm{Lt}$ ), center of the hip joint (Rt and Lt), center of the knee joint (Rt and $\mathrm{Lt}$ ), center of the ankle joint (Rt and Lt), calcaneus (Rt and Lt), metatarsophalangeal (MTP) joint (Rt and $\mathrm{Lt}$ ), toe (Rt and Lt), midpoint between the hip joints, and the iliac crest above the great trochanter (Rt and Lt). For precise digitization of the pelvis, semi-spherical markers were attached to the iliac crest above the great trochanter on the running semi-tights. The x-axis of the global coordinate system corresponded to the long axis of the locomotion, the y-axis corresponded to the mediolateral axis of locomotion, and the $\mathrm{z}$-axis corresponded to the vertical direction. Errors in three-dimensional motion analysis were $\pm 0.006 \mathrm{~m}$ (x-axis), $\pm 0.004 \mathrm{~m}$ (y-axis), and \pm $0.006 \mathrm{~m}$ (z-axis). Data were smoothed using a 3-point moving average method with low-pass filtering at $5 \mathrm{~Hz}$. The walking speeds were calculated from the center of body mass, and their averages are $1.54 \pm 0.18 \mathrm{~m} / \mathrm{s}, 1.99 \pm 0.11 \mathrm{~m} /$ $\mathrm{s}$, and $2.42 \pm 0.12 \mathrm{~m} / \mathrm{s}$ for each target speed.

The variables investigated in the present study were shown in Table 1. Step length was calculated by measuring the linear distance from the heel contact point of the trailing extremity to the heel contact point of the leading extremity. Step length was divided into four components: foot length (FL); TD; RD; and DD. TD ${ }^{7,15,16)}$ is the anterior horizontal distance between the heel of the touch down foot and the center of mass, calculated from body inertia parameters ${ }^{17}$ ) at the moment of foot touch down. RD at the moment of foot release is the anterior horizontal distance between the toe of the releasing foot and the center of mass. The center of mass moves forward during the double stance phase, which is the period from foot touch down to foot release. Displacement of the center of mass during this phase was calculated as DD. Step length was determined as TD + RD + FL - DD. Step length, TD, RD and DD were normalized to the body height of the subject. Walking speed was calculated from anterior displacement of the center of mass over 2 steps. Step frequency was calculated by measuring the reciprocal of step duration between the heel of the touch down foot in two successive steps.

Rotation of the pelvis in the horizontal plane was defined as the angle between the y-axis of the global coordinate system and the line joining both semi-spherical markers on the pelvis. Rotation of the pelvis is commonly referred to in terms of internal and external rotation ${ }^{13,18)}$. In the present study, right leg movement was analyzed as the reference side, and counter-clockwise rotation of the pelvis, as viewed from above, was defined as internal rotation. Internal pelvic rotation thus occurred when the reference (right) side of the pelvis was rotating anteriorly. Pelvic rotation angle was measured at the moment of foot touch down, foot release, and mid-stance. Mid-stance was taken as the moment when the center of body mass reached the toe of the touch down foot.

The angle of the hip joint was defined as the angle between the line from the center of the thorax at the sternum level to the hip joint and the line from the knee joint to the hip joint. The angle of the knee joint was defined as the angle between the line from the hip joint to the knee joint and the line from the ankle joint to the knee joint. The angle of the ankle joint was defined as the angle between the line from the knee joint to the ankle joint and the line from the MTP joint to the ankle joint. Flexed positions of the hip and knee, and the dorsiflexion position of the ankle were defined as positive angles. The angle of the trunk in the sagittal plane was defined as the angle between the line from the hip joint to the center of the thorax at the sternum level and the vertical line.

Least-squares regression analysis was used to fit linear and quadratic polynomials to each data set ${ }^{1)}$. Pearson regression analysis was used to examine whether any of the variables correlated with TD or RD. Values of $p<0.05$ were considered statistically significant. All statistical analyses were performed using SPSS version 11.0 software (SPSS Japan, Japan).

\section{RESULTS}

We plotted each data point for step length, step frequency, TD, DD, and RD as obtained in the motion analysis. Step length and step frequency both showed significant linear increases with walking speed $(r=0.74$, $\mathrm{p}<0.001$ and $\mathrm{r}=0.94, \mathrm{p}<0.001$, respectively). The relationship between TD and walking speed was best approximated by quadratic polynomials $(\mathrm{r}=0.69, \mathrm{p}<0.05)$. TD increased as walking speed rose from $1.3 \mathrm{~m} / \mathrm{s}$ to $1.9 \mathrm{~m} / \mathrm{s}$, then decreased after $1.9 \mathrm{~m} / \mathrm{s}$. RD showed a significant linear increase with walking speed $(r=0.86, p<0.001)$. The 
Table 1. Study variables, and their measurements

\begin{tabular}{|c|c|c|}
\hline Variable & Abbreviation & Measures \\
\hline Step length & & $\begin{array}{l}\text { The linear distance from the heel contact point of the trailing extremity to } \\
\text { the heel contact point of the front extremity caculated by motion analysis }\end{array}$ \\
\hline Step frequency & & $\begin{array}{l}\text { The reciprocal of step duration between the heel of the touch down foot in } \\
\text { two successive steps }\end{array}$ \\
\hline Touch down distance & $\mathrm{TD}$ & $\begin{array}{l}\text { The distance between the heel contact point and center of mass at the } \\
\text { moment of foot touch down }\end{array}$ \\
\hline $\begin{array}{l}\text { Displacement of the center of mass } \\
\text { during double support }\end{array}$ & $\mathrm{DD}$ & Displacement of the center of mass during double foot support phase \\
\hline Release distance & $\mathrm{RD}$ & $\begin{array}{l}\text { The distance between the toe off point and center of mass at the moment of } \\
\text { foot release }\end{array}$ \\
\hline Internal pelvic rotation & IPR & $\begin{array}{l}\text { Pelvic angle when the right side of the pelvis rotated anteriorly in the } \\
\text { horizontal plane at the moment of right foot touch down }\end{array}$ \\
\hline External pelvic rotation & EPR & $\begin{array}{l}\text { Pelvic angle when the right side of the pelvis rotated posteriorly in the } \\
\text { horizontal plane at the moment of right foot release }\end{array}$ \\
\hline Hip flexion or extension angle & & $\begin{array}{l}\text { The angle between the line from the center of the thorax at the sternum level } \\
\text { to the hip joint and the line from the knee joint to the hip joint on the sagittal } \\
\text { plane at the moment of foot touch down and foot release }\end{array}$ \\
\hline Knee flexion or extension angle & & $\begin{array}{l}\text { The angle between the line from the hip joint to the knee joint and the line } \\
\text { from the ankle joint to the knee joint on the sagittal plane at the moment of } \\
\text { foot touch down and foot release }\end{array}$ \\
\hline Ankle plantar flexion angle & Ankle PF & $\begin{array}{l}\text { The angle between the line from the knee joint to the ankle joint and the line } \\
\text { from the MTP joint to the ankle joint at the moment of foot touch down }\end{array}$ \\
\hline
\end{tabular}

relationship between DD and walking speed was best approximated by quadratic polynomials $(\mathrm{r}=0.66, \mathrm{p}<0.05)$. DD increased up to a walking speed of around $2.0 \mathrm{~m} / \mathrm{s}$, but decreased thereafter.

We plotted the pelvic rotation angle at the moment of foot touch down, foot release, and mid-stance as obtained in the motion analysis. The pelvis demonstrated maximum internal rotation at the moment of foot touch down and maximum external rotation at the moment of foot release. Thus, the pelvis thus consistently rotated externally throughout the foot contact period. The relationship between the internal pelvic rotation angle at the moment of foot touch down and the increase in walking speed was best approximated by quadratic polynomials $(\mathrm{r}=0.72, \mathrm{p}<0.005)$. Internal pelvic rotation angle increased with walking speed before plateauing at around $2.0 \mathrm{~m} / \mathrm{s}$. Pelvic rotation angle at the mid-stance was neutral at all walking speeds. External pelvic rotation angle at the moment of foot release with respect to the increase in walking speed was best approximated by quadratic polynomials $(\mathrm{r}=0.74, \mathrm{p}<0.005)$. External pelvic rotation angle increased with walking speed before plateauing at around $2.0 \mathrm{~m} / \mathrm{s}$.

We plotted each data point for the angles of the hip, knee, and ankle joints at the moment of foot touch down and foot release as obtained in the motion analysis. Angles of the hip, knee, and ankle joints at the moment of foot touch down showed no significant change with increasing walking speed. Hip extension angle and ankle plantar flexion angle at the moment of foot release increased linearly with increasing walking speed. The correlation between these variables was significant $(r=0.61, p<0.01$ and $\mathrm{r}=-0.55, \mathrm{p}<0.05$, respectively). However, the knee joint angle at the moment of foot release showed no significant relationship with increasing walking speed. Angles of the trunk in the sagittal plane at the moment of foot contact and foot release showed no significant relationship with walking speed.

TD demonstrated a significant positive correlation with internal pelvic rotation angle $(\mathrm{r}=0.54 ; \mathrm{p}<0.05)$, but no significant association with step length, hip flexion angle, or knee extension angle at the moment of foot touch down (Table 2).

$\mathrm{RD}$ demonstrated a significant positive correlation with step length $(\mathrm{r}=0.50 ; \mathrm{p}<0.05)$, and also with external pelvic rotation angle, hip extension angle, and ankle plantar flexion angle at the moment of foot release $(r=0.56, p<0.05$; $\mathrm{r}=0.61, \mathrm{p}<0.01$; and $\mathrm{r}=0.50, \mathrm{p}<0.05$, respectively) (Table 2 ).

\section{DISCUSSION}

The target walking speeds of $1.3,1.9$ and $2.5 \mathrm{~m} / \mathrm{s}$ in the present study approximately corresponded to preferred, fast and very fast walking speeds, respectively, according to Sekiya and Nagasaki ${ }^{19}$. Walking speed is the product of step length and step frequency, and a linear increase in step length and step frequency with walking speed has been reported $^{1,2,4)}$.

The present study demonstrated the components of step length in relation to walking speed, calculating TD and RD using three-dimensional motion analysis. We then examined the role of pelvic rotation and lower extremity angle on elongation of step length in relation to walking speed. Step 
Table 2. The factors to length the both TD and RD using regression analysis

\begin{tabular}{cccccc}
\hline TD & $\begin{array}{c}\text { Step length } \\
(\mathrm{r}=0.17)\end{array}$ & $\begin{array}{c}\mathrm{IPR}^{*} \\
(\mathrm{r}=0.54)\end{array}$ & $\begin{array}{c}\text { Hip flexion } \\
(\mathrm{r}=0.33)\end{array}$ & $\begin{array}{c}\text { Knee extension } \\
(\mathrm{r}=0.03)\end{array}$ & \\
\hline RD & $\begin{array}{c}\text { Step length* } \\
(\mathrm{r}=0.50)\end{array}$ & $\begin{array}{c}\text { EPR* } \\
(\mathrm{r}=0.56)\end{array}$ & $\begin{array}{c}\text { Hip extension** } \\
(\mathrm{r}=0.61)\end{array}$ & $\begin{array}{c}\text { Knee flexion } \\
(\mathrm{r}=0.36)\end{array}$ & $\begin{array}{c}\text { Ankle PF* } \\
(\mathrm{r}=0.50)\end{array}$ \\
\hline
\end{tabular}

Note. $\mathrm{TD}=$ touch down distance, $\mathrm{RD}=$ release distance, $\mathrm{IPR}=$ internal pelvic rotation, $\mathrm{EPR}=$ external pelvic rotation, $\mathrm{PF}=$ plantar flexion, $\mathrm{NS}=$ no significant. The variables are values at the moment of foot touch down or foot release. *: $<<0.05, * *: p<0.01$ indicate significant in Pearson regression analysis.

length is calculated as TD $+\mathrm{RD}+\mathrm{FL}-\mathrm{DD}$. FL is considered to remain constant at different increasing walking speeds. Studies have reported that an increase in step length with walking speed results from elongation of the TD ${ }^{9-12,15,20)}$. A previous study suggested without providing a rationale that the elongation of step length contributes to moving the front extremity forward. Step length is approximately the base of a triangle formed by the two limbs at the moments of foot touch down and foot release $^{4)}$. Theoretically, the factors that lengthen TD are internal pelvic rotation, hip flexion, and knee extension of the touch down limb ${ }^{9,15,20)}$. In the present study, although step length increased linearly with walking speed, variations in TD and internal pelvic rotation angle as a function of increasing walking speed peaked at around $2.0 \mathrm{~m} / \mathrm{s}$ walking speed, while the hip and knee angles remained constant despite at all increasing walking speeds. Trunk angle in the sagittal plane mainly affected TD, but it did not show any consistent change with increasing walking speed. These results suggest that elongation of TD with walking speed up to around $2.0 \mathrm{~m} / \mathrm{s}$ is caused mainly by increases in the internal pelvic rotation angle. The cause of TD shortening thereafter remains unclear, but most likely involves complex interactions of the pelvis and extremity joints. The present results relating to TD contradict the findings of previous studies ${ }^{9-12,15,20)}$.

DD decreased above a walking speed of around $2.0 \mathrm{~m} / \mathrm{s}$. Decreased double stance duration and increased step frequency are normally accompanied by increasing walking speed. Thus, we suggest that the decreases in DD above 2.0 $\mathrm{m} / \mathrm{s}$ were the result of decreases in double stance duration. RD increased linearly with step length in the present study. Theoretically, factors lengthening RD include the angles of external pelvic rotation, hip extension, knee flexion, and ankle plantar flexion at the moment of foot release. The present results demonstrate that the increase in $\mathrm{RD}$ is caused mainly by increases in hip extension angle and ankle plantar flexion angle at the moment of foot release at walking speeds up to $2.5 \mathrm{~m} / \mathrm{s}$, and also by increases in external pelvic rotation angle at walking speeds below 2.0 $\mathrm{m} / \mathrm{s}$. We suggest that RD is directly related to step length. The linear increase in step length with walking speed was consistent with the linear increase in RD. We confirmed that lengthening the RD is the primary factor by which healthy individual increase step length; Finally, however, we did not investigate the factors to elongating the step length during walking by those with walking disorders.

In conclusion, step length, and step frequency and RD increased linearly with walking speed, RD increased linearly with walking speed, whereas TD and DD did not. Increased step length with increasing walking speed was caused by elongation of $\mathrm{RD}$, mainly resulting from increases in external pelvic rotation, hip extension, and ankle plantar flexion.

Three main limitations of this study warrant consideration. First, data were only obtained from a small number of healthy subjects. Second, differences in motion of the bone and semi-tights may have resulted in measurement errors, as the semi-spherical markers were attached to the semi-tights. Third, the present study examined factors associated with increased step length from the perspective of kinematic parameters. In the future, we hope to examine the elongation of step length at specific torques, with a focus on synthesizing motion analysis and ground reaction force.

\section{REFERENCES}

1) Andriacchi TP, Ogle JA, Galante JO: Walking speed as a basic for normal and abnormal gait measurements. J Biomech, 1977, 10: 261-268.

2) Hirokawa $S$ : Normal gait characteristics under temporal and distance constraints. J Biomed Eng, 1989, 11: 449-456.

3) Vaughan CL: Biomechanical of running gait. Crit Rev Biomed Eng, 1984, 12: $1-48$.

4) Zatsiorky VM, Werner SL, Kaimin MA: Basic kinematics of walking. Step length and step frequency. A review. J Sports Med Phys Fitness, 1994, 34: 109-134.

5) Winter DA: The biomechanics and motor control of human gait. Waterloo: University of Waterloo Press, 1987.

6) Winter DA, Patla AE, Frank JS, et al.: Biomechanical walking pattern changes in the fit and healthy elderly. Phys Ther, 1990, 70: 340-347.

7) Mann R, Sprague P: A kinetic analysis of ground leg during sprint running. Res Q Exerc Sport, 1980, 51: 334-348.

8) Murray MP: Gait as a total pattern of movement. Am J Phys Med, 1967, 46: 290-333.

9) Novacheck TF: Walking, running, and sprinting; A three dimensional analysis of kinematics and kinetics. Instr Course Lect, 1995, 44: 497-506.

10) Perry J: Gait analysis normal and pathological function. America: SLACK, 1992.

11) Huang Y, Meijer OG, Lin J, et al.: The effect of stride length and stride frequency on trunk coordination in human walking. Gait Posture, 2010, 31: $444-449$.

12) Bruijn SM, Meijer OG, van Dieën JH, et al.: Coordination of leg swing, thorax rotations, and pelvis rotations during gait: The organisation of total body angular momentum. Gait Posture, 2008, 27: 455-462.

13) Inman VT, Ralston HJ, Todd F: Human walking. Baltimore: Williams and Wilkins, 1981, pp 1-61.

14) Winter DA: Biomechanics and motor control of human movement, 2nd ed. 
Hoboken: John Wiley \& Sons, 1990.

15) Novacheck TF: The biomechanics of running. Gait Posture, 1998, 7: 77-95.

16) Slocum DB, Bowerman W: The biomechanics of running. Clin Orthop 1962, 23: 39-45.

17) Matsui H: Determination of center of gravity of human body in various posture. I. Center of gravity calculated with simplified mass values. Jpn J Phys Educ, 1956, 56: 65-76.
18) Ounpuu S: The biomechanical of walking and running. Clin Sports Med, 1994, 13: 843-863.

19) Sekiya N, Nagasaki H: The invariant relationship between step length and step rate during free walking. J Hum Mov Stud, 1996, 30: 241-257.

20) Schache AG, Bennell KL, Blanch PD, et al.: The coordinated movement of the lumbo-pelvic-hip complex during running: a literature review. Gait Posture, 1999, 10: 30-47. 